\title{
WHEN IS A NONLINEAR VISCOELASTIC MATERIAL HYPERELASTIC?*
}

\author{
BY DAVID W. REYNOLDS \\ (University College Dublin)
}

1. Introduction. This note presents two different characterizations, in terms of work, of hyperelastic materials within the wider class of nonlinear viscoelastic ones. Here a viscoelastic material is regarded as one in which the stress depends continuously on strain history and has a mild form of fading memory. If the stress equals the gradient of a potential evaluated at the present strain, the material is hyperelastic. In such a material, the stress depends on the strain history only through its present value.

The first characterization is an improvement upon a theorem of Day [1] (cf. [2] for another theorem of this type). It is that if a viscoelastic material does no recoverable work in closed continuations of strain histories, then the material must be hyperelastic. The other result is that a viscoelastic material, which is compatible with thermodynamics and almost conservative, is also hyperelastic. The meaning of these terms is given later.

The proofs involve the construction of a potential for the stress. It is therefore not surprising that ideas found in Day [1,3] on the construction of entropy functions for simple materials with fading memory are employed here.

2. Preliminaries. Let us now proceed with a more precise treatment of these ideas. The terminology chosen is meant to suggest applications in mechanics, though the theorems are also relevant to thermodynamics.

Let $\mathscr{U}$ be an open, connected subset of a real finite dimensional inner product space $\mathscr{V}$. In mechanics, $\mathscr{V}$ is usually taken to be the space of endomorphisms of a threedimensional Euclidean space, and $\mathscr{U}$ the subset of those with strictly positive determinant. In this case the inner product and norm on $\mathscr{V}$ are given by

$$
\alpha \cdot \beta=\operatorname{trace}\left(\alpha \beta^{T}\right), \quad|\alpha|=\sqrt{\alpha \cdot \alpha},
$$

respectively, where $\beta^{T}$ is the transpose of $\beta$.

A strain process is defined to be a continuous, piecewise smooth function $\varepsilon:[0, u] \rightarrow \mathscr{U}$ ("smooth" is used throughout as a synonym for "continuously differentiable"). The number $u$ is called the duration of the process, and the values

$$
\varepsilon^{i}=\varepsilon(0), \quad \varepsilon^{f}=\varepsilon(u)
$$

are referred to as the initial and final values of the process. If $\varepsilon^{i}=\varepsilon^{f}$, the process $\varepsilon$ is termed closed. The process which has constant value $\alpha$ and duration $u$ is denoted by $\alpha_{(u)}$, i.e.

$$
\alpha_{(\mathrm{u})}(\mathrm{t})=\alpha,
$$

* Received March 10, 1982. The author is grateful to Dr. W. A. Day and Professor M. E. Gurtin for valuable discussions on this paper. This work was supported by the U.S. Army Research Office. 
for all $t$ in $[0, u]$. Let $\varepsilon$ and $\xi$ be two processes of duration $u$ and $v$ respectively. If $\xi^{i}=\varepsilon^{f}$, a new process $\varepsilon \circ \xi$ can be constructed by setting

$$
(\varepsilon \circ \xi)(t)= \begin{cases}\varepsilon(t), & 0 \leq t \leq u, \\ \xi(t-u), & 0 \leq t \leq u+v .\end{cases}
$$

The history of a strain process $\varepsilon$ up to time $t$ in $[0, u]$ is the mapping $\varepsilon^{t}:[0, \infty) \rightarrow \mathscr{U}$ given by

$$
\varepsilon^{t}(s)= \begin{cases}\varepsilon(t-s), & 0 \leq s \leq t, \\ \varepsilon(0), & s \geq t,\end{cases}
$$

where $u$ denotes the duration of $\varepsilon$. It is easily seen that the collection of all histories corresponding to all strain processes coincides with $\mathscr{H}$, the set of continuous piecewise smooth functions $h:[0, \infty) \rightarrow \mathscr{U}$, which are constant outside a compact interval. For this reason, elements of $\mathscr{H}$ are called strain histories. The history $\alpha^{*}$ with constant value $\alpha$ in $\mathscr{U}$ is a simple example of a strain history. A process $\varepsilon$ is termed a closed continuation of $h$ in $\mathscr{H}$ at time $t$ if $\varepsilon^{t}=h, \varepsilon(t)=\varepsilon^{f}$. The metric

$$
d\left(h_{1}, h_{2}\right)=\sup \left\{\left|h_{1}(s)-h_{2}(s)\right|: s \geq 0\right\}
$$

endows $\mathscr{H}$ with a topology. It is finer than those introduced by Coleman and Noll [4] and Coleman and Mizel [5] to model fading memory properties of functionals defined on history spaces.

By a stress response function we mean a continuous mapping $\sigma: \mathscr{H} \rightarrow \mathscr{V}$. The continuity requirement expresses the notion that stresses corresponding to strain histories which are uniformly close must themselves be close. The function $t \mapsto \sigma\left(\varepsilon^{t}\right)$ can be interpreted as describing the evolution of the Piola-Kirchoff stress at a material point where the deformation gradient is given by $t \mapsto \varepsilon(t)$. A simple case of a stress response function is provided when $\sigma$ is hyperelastic; i.e. there is a smooth potential $\phi: \mathscr{U} \rightarrow \mathbb{R}$ such that

$$
\sigma(h)=\nabla \phi(h(0)),
$$

for all $h$ in $\mathscr{H}$. It is easily proved that a stress response function $\sigma$ satisfies:

(i) $t \mapsto \sigma\left(\varepsilon^{t}\right)$ is continuous, for each strain process $\varepsilon$;

(ii) the associated equilibrium stress response function $\sigma^{*}: \mathscr{U} \rightarrow \mathscr{V}$ is continuous, where

$$
\sigma^{*}(\alpha)=\sigma\left(\alpha^{*}\right),
$$

for all $\alpha$ in $\mathscr{U}$.

(i) eliminates from consideration some materials of the differential type, among them viscous fluids, which would otherwise provide counter-examples to Theorem 1 and in some cases Theorem 2.

The work done (by a stress $\sigma$ ) in a strain process $\varepsilon:[0, u] \rightarrow \mathscr{U}$ is given by the formula

$$
w(\varepsilon)=\int_{0}^{u} \sigma\left(\varepsilon^{s}\right) \cdot \dot{\varepsilon}(s) d s,
$$

where $\dot{\varepsilon}$ denotes the derivative of $\varepsilon$. It should be pointed out that the stress response function can also be viewed as giving the values of the generalized stress (cf. e.g. [3, p. $32]$ ), in which case $-w$ is the Clausius integral.

The properties of stress response functions are insufficient to prove our theorems, nor 
do they include any kind of fading memory property. Let us adopt a concept of fading memory introduced in [1]. For the purposes of this note, a stress response function is called viscoelastic if it enjoys fading memory in the following sense ${ }^{1}$ :

$$
w\left(\varepsilon \circ \varepsilon_{(u)}^{f} \circ \varepsilon\right) \rightarrow w(\varepsilon)+w(\xi)
$$

as $u \rightarrow \infty$, for each pair of processes $\varepsilon, \xi$ with $\varepsilon^{f}=\xi^{i}$.

The following terminology helps us to state the results succinctly. The maximum work recoverable from a strain history is defined by (cf. [7], [1, p. 9])

$$
m(h)=\sup \left\{-\int_{t}^{u_{\varepsilon}} \sigma\left(\varepsilon^{s}\right) \cdot \dot{\varepsilon}(s) d s: \varepsilon \text { is closed continuation of } h(\text { at } t)\right\},
$$

where $u_{\varepsilon}$ is the duration of $\varepsilon$. A stress function is said to:

(i) be compatible with thermodynamics $[c f .[8,3])$ if $w(\varepsilon) \geq 0$ for every closed process $\varepsilon$

(ii) be conservative if $w(\varepsilon)=0$ for all closed processes $\varepsilon$;

(iii) be almost conservative if, for every process $\varepsilon$ and $\kappa>0$, there is a process $\eta$ with $\eta^{i}=\varepsilon^{f}, \eta^{f}=\varepsilon^{i}$ such that

$$
w(\varepsilon)+w(\eta)<\kappa ;
$$

(iv) never recover work if $m(h)=0$ for all $h$ in $\mathscr{H}$.

Of course, a conservative viscoelastic stress function is almost conservative, for if $\varepsilon$ and $\eta$ are as in (iii), $\varepsilon \circ \varepsilon_{(t)}^{f} \circ \eta$ is closed, so that

$$
w\left(\varepsilon \circ \varepsilon_{(t)}^{f} \circ \eta\right)=0 .
$$

By letting $t \rightarrow \infty$ and using the fading memory of the stress, it follows that

$$
w(\varepsilon)+w(\eta)=0 .
$$

\section{Statement and proof of results.}

THEOREM 1. A viscoelastic stress function is hyperelastic if and only if it never recovers work.

This extends Theorem 3 of [1] to a less restrictive class of stress response functions. Day's theorem has the additional hypotheses that the stress behave elastically in both slow and fast processes (see [1, p. 7]) and he employs a different continuity assumption. In [5], after characterizing the influence measures $\mu$ which endow the history space $L_{\mu}^{p}([0$, $\infty), \mathscr{U}$ ) with certain physical properties, additional restrictions had to be placed on $\mu$ to ensure that continuous functions $\sigma: L_{\mu}^{p}([0, \infty), \mathscr{U}) \rightarrow \mathscr{V}$ have the retardation property ${ }^{2}$ This suggests that it is desirable to remove the hypotheses of elastic behaviour in slow and fast processes.

THEOREM 2. A viscoelastic stress function is hyperelastic if and only if it is almost conservative and compatible with thermodynamics.

Due to the remark at the end of Sec. 2, this theorem has the immediate

${ }^{1}$ Cf. [1, p. 6], [3, p. 36]. Theorems 1 and 2 are true under the weaker assumption that $A 2$ of [6] holds with $-w$ replacing $I$.

${ }^{2}$ The hypotheses of the retardation theorem in [5] are essentially those used in [4]. 
COROllary. A viscoelastic stress function is hyperelastic if and only if it is conservative.

Proof of Theorem 1. The necessity being evident, only the sufficiency is proved here. Let $\sigma$ be a viscoelastic stress function which never recovers work. Let $h$ be an arbitrary strain history. Then there is a process $\varepsilon$ of duration $v$ such that $\varepsilon^{v}=h$. Let $\beta$ be in $\mathscr{V}$. Because $\alpha=h(0)$ is an element of the open set $\mathscr{U}$, there is a $u>0$ such that $\alpha+\beta s$ is in $\mathscr{U}$ for all $s$ in $[0, u]$. Define a strain process $\xi:[0, u] \rightarrow \mathscr{U}$ by

$$
\xi(s)=\alpha+\beta s,
$$

for each $s$ in $[0, u]$. Also, let $\xi_{n}$ be the restriction of $\xi$ to $\left[0, u_{n}\right]$, where $\left(u_{n}\right)$ is any strictly decreasing sequence converging to zero, with $u_{1}=u$.

Since $\varepsilon \circ \xi_{n} \circ\left(\xi_{n}^{f}\right)_{(t)} \circ \bar{\xi}_{n}$ is a closed continuation of $h$, the assumption that $\sigma$ never recovers work implies that

$$
w\left(\varepsilon \circ \xi_{n} \circ\left(\xi_{n}^{f}\right)_{(t)} \circ \bar{\xi}_{n}\right)-w(\varepsilon) \geq 0,
$$

for all $n=1,2, \ldots$ and $t>0$. Here the process $\bar{\xi}_{n}:\left[0, u_{n}\right] \rightarrow \mathscr{U}$ is defined by

$$
\bar{\xi}_{n}(s)=\xi_{n}\left(u_{n}-s\right) \text {. }
$$

Let $t \rightarrow \infty$ and appeal to the fading memory property to deduce that

$$
w\left(\varepsilon \circ \xi_{n}\right)-w(\varepsilon)+w\left(\bar{\xi}_{n}\right) \geq 0,
$$

or

$$
\int_{v}^{v+u_{n}} \sigma\left[(\varepsilon \circ \xi)^{s}\right] \cdot \dot{\xi}(s) d s+\int_{0}^{u_{n}} \sigma\left(\bar{\xi}_{n}^{s}\right) \cdot \bar{\xi}_{n}(s) d s \geq 0 .
$$

Since $d\left(\overline{\xi_{n}^{s}}-\alpha^{*}\right) \|=u_{n}|\beta| \rightarrow 0$ uniformly in $s$ as $n \rightarrow \infty, \sup _{s} \geqslant 0\left|\sigma\left(\bar{\xi}_{n}^{s}\right)-\sigma\left(\alpha^{*}\right)\right| \rightarrow 0$ as $n \rightarrow \infty$, and therefore

$$
\frac{1}{u_{n}} \int_{0}^{u_{n}}\left[\sigma\left(\bar{\xi}_{n}^{s}\right)-\sigma\left(\alpha^{*}\right)\right] \cdot \beta d s \rightarrow 0
$$

as $n \rightarrow \infty$. Hence, dividing (1) through by $u_{n}$ and taking the limit as $n \rightarrow \infty$ gives

$$
\left\{\sigma(h)-\sigma^{*}(h(0))\right\} \cdot \beta \geq 0 .
$$

Since this inequality holds for arbitrary $\beta$ in $\mathscr{V}$,

$$
\sigma(h)=\sigma^{*}(h(0))
$$

for all $h$ in $\mathscr{H}$, i.e. $\sigma$ is elastic.

The elasticity of $\sigma$ and our hypothesis that $\sigma$ recovers work imply that

$$
\int_{0}^{u} \sigma^{*}[\varepsilon(s)] \cdot \dot{\varepsilon}(s) d s \geq 0
$$

for every closed process $\varepsilon:[0, u] \rightarrow \mathscr{U}$. It is a standard result (e.g. $[3$, pp. 8, 9]) that this implies the existence of a smooth potential $\phi: \mathscr{U} \rightarrow \mathbb{R}$ such that

$$
\sigma^{*}=\nabla \phi
$$

It follows from (2) and (3) that $\sigma$ is hyperelastic.

Proof of Theorem 2. Again only the sufficiency is proved. Suppose that $\sigma$ is an almost conservative stress function, which is compatible with thermodynamics. Let $h$ be in $\mathscr{H}$. As 
before, there is a process of duration $v$ such that $\varepsilon^{v}=h$. Let $\xi$ be an arbitrary closed process with $\xi^{i}=\xi^{f}=h(0)$. Let $\kappa>0$. Since $\sigma$ is almost conservative there is a process $\eta$ with $\eta^{i}=\varepsilon(v)=h(0), \eta^{f}=\varepsilon(0)$, and

$$
w(\varepsilon)+w(\eta)<\kappa .
$$

For every $t \geq 0, \varepsilon \circ \xi \circ \xi_{(t)}^{f} \circ \eta$ is closed. Compatibility with thermodynamics implies that

$$
w\left(\varepsilon \circ \xi \circ \xi_{(t)}^{f} \circ \eta\right) \geq 0
$$

for all $t \geq 0$, and hence

$$
w(\varepsilon \circ \xi)+w(\eta) \geq 0,
$$

due to the fading memory of the stress. By (4) and (5)

$$
w(\varepsilon \circ \xi)-w(\varepsilon) \geq-w(\varepsilon)-w(\eta) \geq-\kappa .
$$

Since $\kappa$ is an arbitrary positive number, it follows that

$$
w(\varepsilon \circ \xi)-w(\varepsilon) \geq 0 .
$$

But the left-hand side is the work done in an arbitrary closed continuation $\varepsilon \circ \xi$ of $h$; thus $m(h)=0$ for all $h$ in $\mathscr{H}$. The result can now be concluded from Theorem 1 .

\section{REFERENCES}

[1] W. A. Day, Thermodynamics based on a work axiom, Arch. Rational Mech. Anal. 31, 1-34 (1968)

[2] W. A. Day, When is a linear viscoelastic material elastic? Mathematika 18, 134-137 (1971)

[3] W. A. Day, The thermodynamics of simple materials with fading memory, Springer Tracts in Natural Philosophy, Springer-Verlag, Berlin, 1972

[4] B. D. Coleman and W. Noll, An approximation theorem for functionals, with applications in continuum mechanics, Arch. Rational Mech. Anal. 6, 355-370 (1960)

[5] B. D. Coleman and V. J. Mizel, Norms and semi-groups in the theory of fading memory, Arch. Rational Mech. Anal. 23, 87-123 (1966)

[6] W. A. Day, A theory of thermodynamics for materials with memory, Arch. Rational Mech. Anal. 34, 85-96 (1969)

[7] S. Breuer and E. T. Onat, On recoverable work in linear viscoelasticity, Z. Angew. Math. Phys. 15, 12-21 (1964)

[8] B. D. Coleman, Thermodynamics of materials with memory, Arch. Rational Mech. Anal. 17, 1-46 (1964) 